\title{
PTEN Gene Inactivation
}

National Cancer Institute

\section{Source}

National Cancer Institute. PTEN Gene Inactivation. NCI Thesaurus. Code C36692.

A molecular genetic abnormality indicating the presence of an inactivating mutation in the PTEN gene on chromosome 10q23. 